\title{
Design micro-piezoelectric actuated gripper for medical applications
}

\author{
Musaddiq Al Ali a, , Muazez Al Alib, Amjad Y. Sahib ${ }^{c}$,Rajaa S. Abbas ${ }^{\mathrm{d}}$ \\ ${ }^{a}$ Hiroshima University, 1-3-2 Kagamiyama, Higashi-Hiroshima City, 739-8511, Japan \\ ${ }^{\mathrm{b}}$ Al Ayen University, Thi Qar Province, Niel St., Nasiriyah, Iraq \\ 'University of Wasit, Wasit Province, Hay Al Rabee, Kut, Iraq \\ ${ }^{\mathrm{d} A 1}$ Mamon University College, 14 Ramadan St., Baghdad, Iraq
}

*Corresponding Author: mosadeq007@yahoo.com

\begin{abstract}
Designing micro electro-mechanical systems based gripper is challenging task specially with introducing accurate and tactile gripping. Tactile gripping is important with biological and medical application. In this work, single structure mechanism of piezoelectric materials is designed using topology optimization to be a micro gripper. Topology optimization is done in MATLAB programming environment. Model is tested in COMSOL. The models showed good opening and closing action actuated by applying electric potential.
\end{abstract}

Keywords: MEMS, Piezoelectric, topology optimization.

\section{Introduction}

Due to the advancement of medical imaging process and real-time radiography, it is possible to operate remotely, without the doctor physically do the operation, instead, the doctor uses highly precision robotic devices to conduct the operation $^{(1,2)}$. In some parts of human body such as nerval systems; the precision is highly important. The prison problem can be controlled by decreasing the size of the robotic arm to the suitable minimum size. Still, the motor vibration and transmission can cause unnecessary movement, endangering the patient. The task of designing small robotic hand with low vibration actuation method is the goal of this work. Micro and non-robotic arms application goes beyond medical applications, so such mechanisms can make the manufacturing of Nano devices, and electronics, simpler. Nanoscale crafted electronics are used in consumer computers, and smartphones. Introducing considerable simple design, with high precision nanorobotics can be a useful tool to decrease the scale of nanoscale crafting. For example, moving atoms using Scanning Tunneling Microscope (STM) can be a systematic production process for Nano crafting ${ }^{(3,4)}$.

Topology optimization (TO) be designed method based on a mathematical scheme to introduce non-existence material within design space to get designer properties such as light weight or certain mechanical properties. Ole Sigmund at $1997^{(5)}$, studied compliant mechanisms stress minimization by constraining allowable displacement of the mechanism itself. Nishiwaki et al at 1998 (6) used homogenization method as topology optimization scheme for designing half gripper, with scissor like applied actuation forces. Pedersen et al at $2001{ }^{(7)}$ studied large displacement compliant mechanism. Sensitivity and scheme based on large displacement non-linear finite elements.

Piezoelectric material using as actuator was presented practically, first by Williams and Brown et at $1940^{(8)}$. They introduced first piezoelectric motor. Followed by Lavrinenko and Nekrasov 1965. At 1987, D.W. Pohl (9) introduce piezoelectric inertia actuator. The Same year, Anders et al ${ }^{(10)}$ developed micro positioning devices for scanning tunneling microscope (STM). At 1990 Renner et al ${ }^{(11)}$ developed antigravity linear translation device using piezoelectric induced slip stick motion. A vertical/ horizontal piezoelectric inertial slider was developed by Blackford et al (12) at 1992. Bergander et al ${ }^{(13)}$, at 2002, studied piezoelectric actuators mechanisms for high precision motion. Small size piezoelectric robotic can be considered as Micro ElectroMechanical Systems (MEMS). MEMS have several challenges which also the advantages of such systems. First is the spacial challenge (small size manufacturing). So, the process of making small complex linking mechanisms, gears, 
etc. is a difficult task, add to that the cost factor, so for high quality micro and Nano devices, even with relatively simple set of gears, moving joints and links, needs highly specialized processes and expensive machines. In this work, we introduce, compliance mechanism as a way of design simple and one-piece mechanism that achieve the task such as clamping or grapping by applying currents on it. Within the advancement in material processing, many techniques been advanced for cheaper micro and Nano structure manufacturing such as lithography and $\mathrm{CVD}^{(14,15)}$. For example, Toledo et al ${ }^{(16)}$ at 2017 successfully built compliant mechanism using Silicon on insulator base actuator. In this paper, we designed robotic grabbing mechanism using topology optimization of a piezoelectric material (Zinc Oxide) micro and nanoscale.

\section{Topology optimization designing process}

Topology optimization was been developed from Maxwell dilemma of optimal weight truss design, by Michell ${ }^{(17)}$. Himp ${ }^{(18)}$ studied strain field by adopting slip line, which at that time was regarded as an important aspect of plastic deformation implementations. Drucker et al (19) applied constant dissipation per unit volume as their study to stress strain fields and strain energy. Chan ${ }^{(20)}$ study the optimization of static stability of truss structure by developing a technique to determine topographic based strain filed. Charrett and Rozvany ${ }^{(21)}$ adopted Prager - shield implementation in order to find optimal design criterion considering rigid-perfectly plastic systems under multiple loading. Rozvany and Prager ${ }^{(22)}$ studied optimal design of grillage like continua. Their approach was spatial distribution within confined grillage units. Rossow and Taylor ${ }^{(23)}$ used finite element method as a numerical solution to find the optimum thickness of variable thickness sheets. Potential energy for the elastic sheet in-plane stress assumption was addressed. By introducing holes into plate structure, this work founded shape optimization. Cheng and Olhoff ${ }^{(24)}$ implement finite element method as a numerical solution to optimize the thickness of annual plate with stiffened like approach. Bendsoe ${ }^{(25)}$ studied Solid Isotropic Material with Penalization (SIMP) based on Taylors work. Solid Isotropic Material with Penalization (SIMP) is a scheme apply for discretized design domain to find smooth optimal structure.in which, material properties set to be constant for the discretized domain, however, the existence of building block which is set in what so-called density function composed of material existence $\rho$ of conditioned power multiply by mechanical properties.

\section{Electro elasticity of Piezoelectric structure}

Piezoelectric materials are anisotropic dielectrics. electricity is varying with external electric and/or force field that is applied to the piezoelectric structure. It forms an electric dipole. Because of dielectric polarization, different charges react positively proportional to the electric field that applied. The polarization can be formulated in terms dipole moments. When a piezoelectric force field act on the piezoelectric material, the topology of the atomic structure of the crystal deviate in a way creating effective dipoles, and a dipole moment is formed. And because of Anisotropic properties, when a piezoelectric material is loaded electrically then the electrical dipoles appear only after electrical or mechanical load. Electro-elasticity deals with such phenomenon ${ }^{(26)}$. The piezoelectric effect is presented as the density electric moment $(\mathrm{P})^{(27)}$ as shown in equation

$$
P=\sum \kappa_{0} \chi_{i j} E_{j}+d \boldsymbol{\sigma}
$$

Here, $\mathrm{d}$ is the piezoelectric modulus associated to stress tensor. Related strain be

$$
\varepsilon=d^{T} E+c^{-1} \sigma
$$

The electromechanical coupling can be derived from Maxwell's. the total strain energy of the electromechanical system is given as

$$
\Pi=\frac{1}{2} C_{i j k} \varepsilon_{i j} \varepsilon_{k l}+d^{T} \varepsilon
$$

The derivative of equation 3 in terms of strain gives the designated stress, and the derivative in terms of electric field vector gives the component of electrically induced displacement.

\section{Case studies}

\subsection{Design procedure}

Topology optimization is implemented in MATLAB programming environment. A code written in MATLAB for 4-node square element adopting SIMP method topology optimization. 


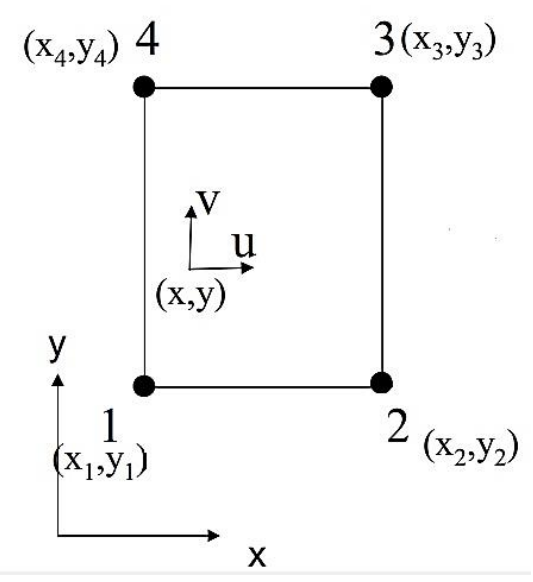

Fig. 1 2-D element aspects

The design procedure is two stage problem, first, the design domain build in MATLAB and set to be topology optimization problem. After getting feasible design from MATLAB. The design be imported to COMSOL to increase mesh resolution and use high order mesh. Mechanical simulation of the problem is set. From the movements of the areas around input load point, the electric wiring for step 3 will be set, i.e. the links near the input loadings are moving in inward or outward, so the electric potential will be reverse on them to set the desire movement of the jaws. As shown in Figure (2)

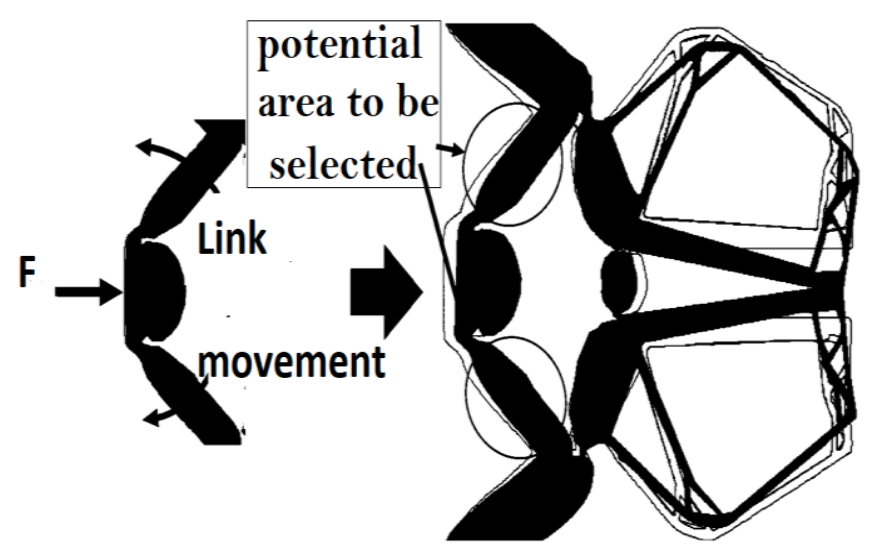

Fig. 2 Micro gripper design principal

The argument of electrode positioning methodology is that, for topology optimization solution, the input force will be transmitted through the links to do the jaw closing or opening, so the induced stress using electric potential difference on those links will assure keeping the same desire opening and closing movement for the final piezoelectric mechanism.

\subsection{Biological application (Micro grippers)}

Two cases study were selected of grabber, Gripper (A) is a micro size grabber (362 by 362 ) micrometer of (43) micrometer opening; and the second case is micro size grabber (Gripper B) of (940 by 940) nanometer of small opening (104) nanometer as shown in Figure 3. The size of the grabber was chosen for the purpose of graph cancer tumors for in vivo photodynamic therapy ${ }^{(28-30)}$ (PDT) using diode laser at wavelength $(650 \mathrm{~nm})$ with methylene blue. Such gripping arm can be mounted on a wire spring guided fiberoptic, then use the gripper to grip the cancer cluster to increase laser irradiance of the affected cells and limit irradiance of the sounded tissue as shown in Figure 4.

Design material is Zinc oxide. Zinc oxide has been used for the medical purpose in dentistry ${ }^{(31)}$ and prosthetic parts. It has also been used as food additive. The final design of the grippers been shown in Figure 5.

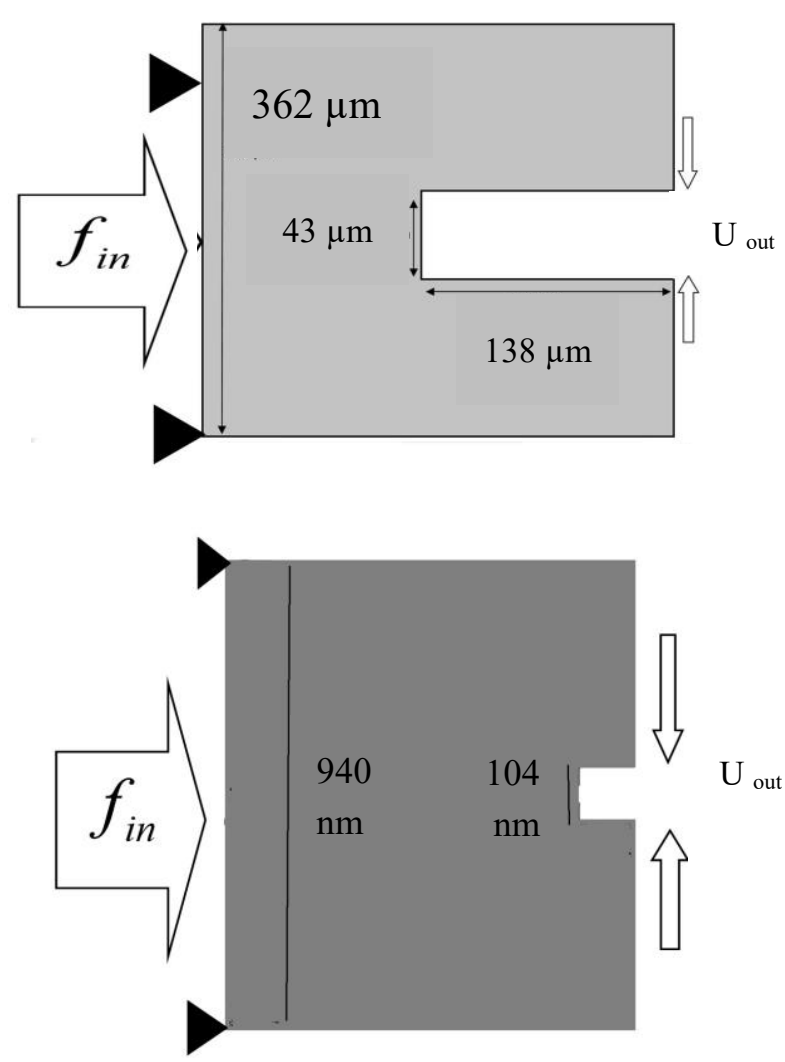

Fig. 3. Design Domain for micro grippers 


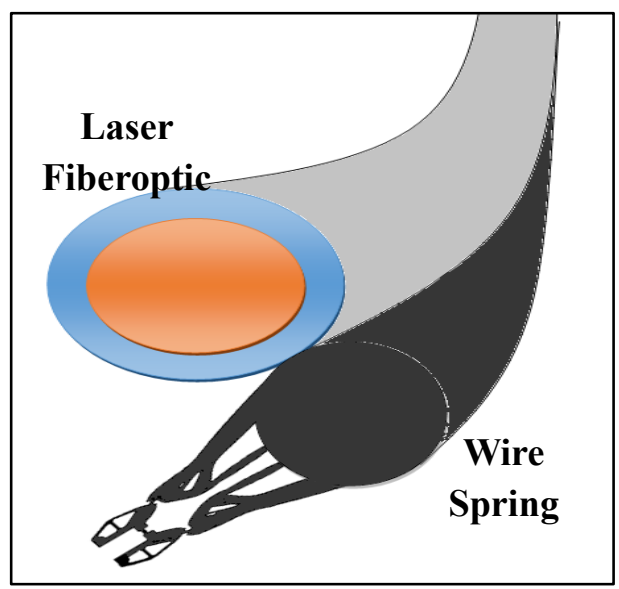

Fig. 4. Gripper mounted in laser therapy apparatus

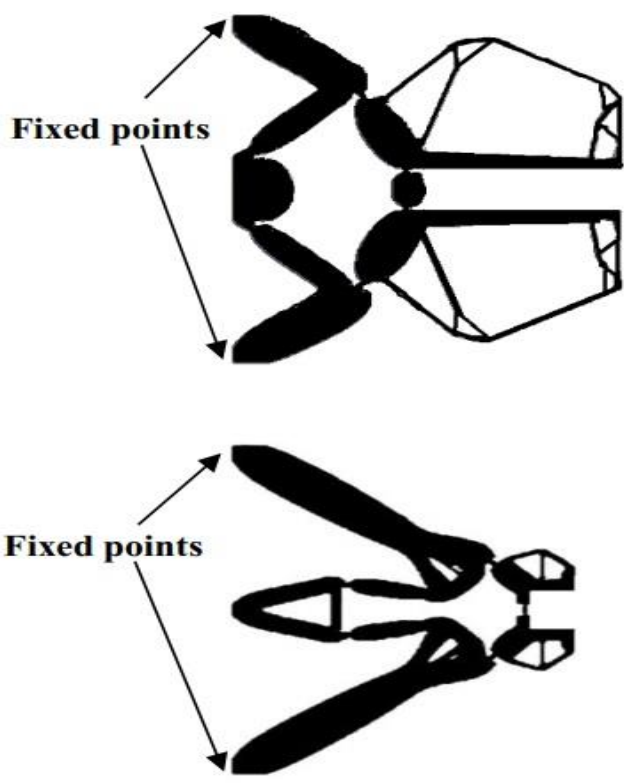

Fig. 5. Topology optimization design of Micro gripper

The second step is import the designs from MATLAB to COMSOL and setup the electric wiring simulation and apply the mechanical boundary conditions as shown in Figure 6. Ground is located on the fixed point of the mechanism. The region above and below the central moving mass will wired with different voltage polarity.

The third step is applying electric boundary conditions. The electric potential varies within the period $(-4,4)$ volts as an open load/close for the gripper. To close the hand, we used the same potential but with reverse polarity. Operating gripper (A) with applying electricity is shown inFigure 7. Figure 8, shows micro gripper (B) in closing and opening mode. To control closing of the micro gripper, small change in potential will suffice. Figure 9 shows good control of displacement per potential change

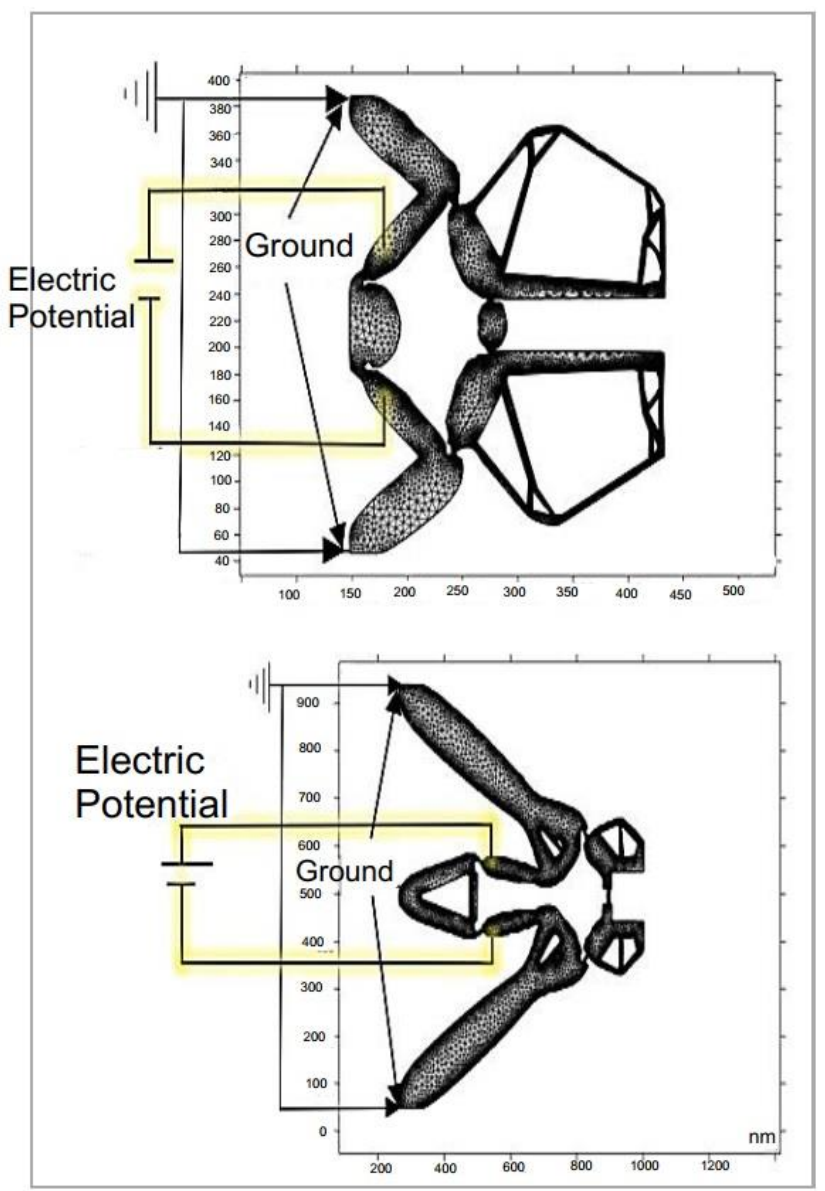

Fig. 6. Electric circuit of piezoelectric micro gripper

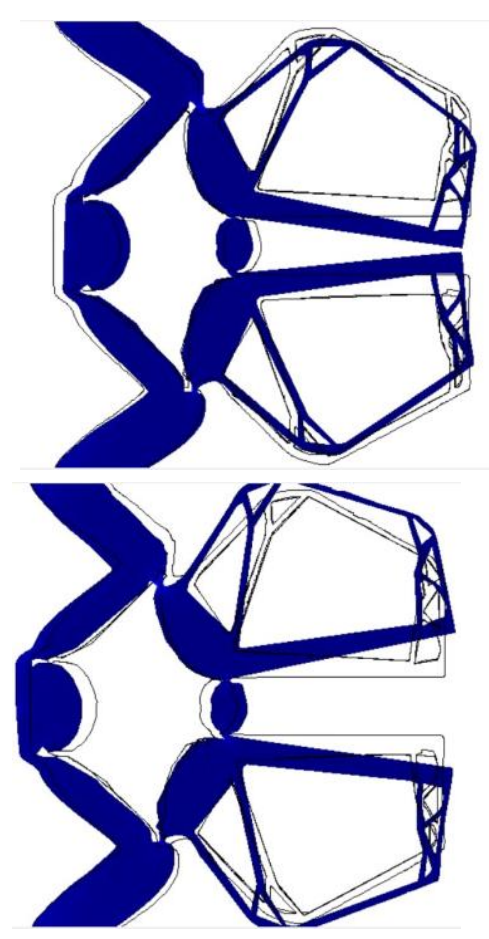

Fig. 7. Micro gripper (A) in fully opening and fully closing mode actuated by piezoelectric action with applied current 


\section{Conclusions}

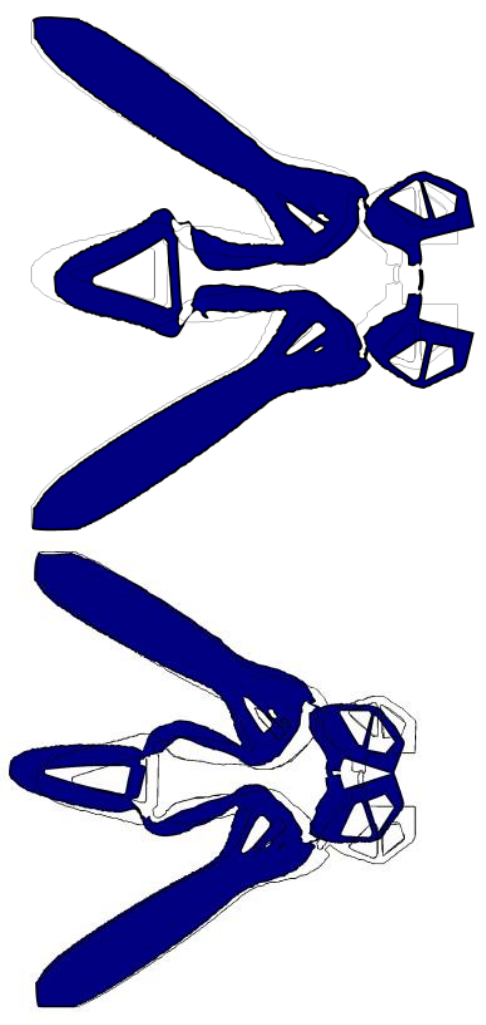

Fig. 8. Micro gripper (B) in fully opening and fully closing mode actuated by piezoelectric action with applied current

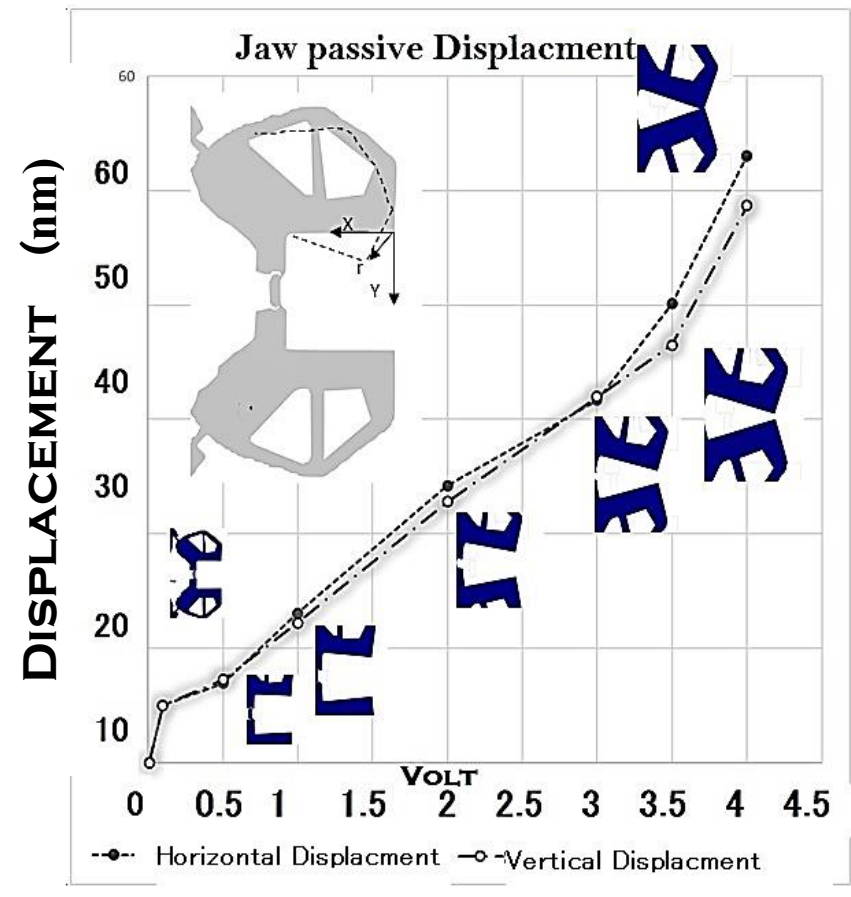

Fig. 9. Closing spacing versus applied potential for micro gripper.
Topology optimization shows good results of designing single structure that can-do movement by applying action in desired points. Compliant mechanisms that is designed using topology optimizations, have no bearing, and do not need complicated links attachment, so it eases the way of making. Making compliant mechanism of piezoelectric will offer self-actuation by applying potential, so it will not need complex mechanism to derive and apply forces. The simulation shows good gripping control by changing the value of the applied potential as shown in Figure 8.

\section{References}

(1) P. Dario, E. Guglielmelli, B. Allotta, and M. C. Carrozza, "Robotics for medical applications," IEEE Robotics \& Automation Magazine, vol. 3, no. 3, pp. 44-56, 1996.

B. J. Nelson, I. K. Kaliakatsos, and J. J. Abbott, "Microrobots for minimally invasive medicine," Annual review of biomedical engineering, vol. 12, pp. 55-85, 2010.

(3) A. Kühnle, G. Meyer, S. Hla, and K.-H. Rieder, "Understanding atom movement during lateral manipulation with the STM tip using a simple simulation method," Surface Science, vol. 499, no. 1, pp. 15-23, 2002.

(4) P. Avouris, "Manipulation of matter at the atomic and molecular levels," Accounts of chemical research, vol. 28, no. 3, pp. 95-102, 1995.

(5) O. Sigmund, "On the design of compliant mechanisms using topology optimization," Journal of Structural Mechanics, vol. 25, no. 4, pp. 493-524, 1997.

(6) S. Nishiwaki, M. I. Frecker, S. Min, and N. Kikuchi, "Topology optimization of compliant mechanisms using the homogenization method," 1998.

(9) D. Pohl, "Sawtooth nanometer slider: A versatile low voltage piezoelectric translation device," 
Surface Science, vol. 181, no. 1-2, pp. 174-175, 1987.

(10) M. Anders, M. Thaer, and C. Heiden, "Simple micropositioning devices for STM," Surface Science, vol. 181, no. 1-2, pp. 176-182, 1987.

(11) C. Renner, P. Niedermann, A. Kent, and O. Fischer, “A vertical piezoelectric inertial slider," Review of scientific instruments, vol. 61, no. 3, pp. 965-967, 1990.

(12) B. Blackford, M. Jericho, and M. Boudreau, "A vertical/horizontal two - dimensional piezoelectric driven inertial slider micropositioner for cryogenic applications," Review of scientific instruments, vol. 63, no. 4, pp. 2206-2209, 1992.

(13) A. Bergander, J.-M. Breguet, and R. Clavel, "Micropositioners for microscopy applications and microbiology based on piezoelectric actuators," Journal of Micromechatronics, vol. 2, no. 1, pp. 6576, 2002.

(14) V. Axelrad, and M. C. Smayling, "16nm with $193 \mathrm{~nm}$ immersion lithography and double exposure." pp. 764109-764109-6.

(15) K. Kim, Y. Sun, R. M. Voyles, and B. J. Nelson, "Calibration of multi-axis MEMS force sensors using the shape-from-motion method," IEEE Sensors Journal, vol. 7, no. 3, pp. 344-351, 2007.

(16) J. Toledo, V. Ruiz-Díez, A. Diaz-Molina, D. Ruiz, A. Donoso, J. C. Bellido, E. Wistrela, M. Kucera, U. Schmid, and J. Hernando-García, "Design and Characterization of In-Plane Piezoelectric Microactuators." p. 19.

(17) A. G. M. Michell, "LVIII. The limits of economy of material in frame-structures," The London, Edinburgh, and Dublin Philosophical Magazine and Journal of Science, vol. 8, no. 47, pp. 589-597, 1904.

(18) W. S. Hemp, Theory of structural design, College of Aeronautics Cranfield, 1958.

(19) D. C. Drucker, and R. Shield, Design for Minimum Weight, BROWN UNIV PROVIDENCE RI, 1956.

(20) A. Chan, The design of Michell optimum structures, College of Aeronautics Cranfield, 1960.

(21) D. Charrett, and G. Rozvany, "Extensions of the Prager-Shield theory of optimal plastic design," International Journal of Non-Linear Mechanics, vol. 7, no. 1, pp. 51-64, 1972.

(22) G. Rozvany, and W. Prager, "Optimal design of partially discretized grillages," Journal of the Mechanics and Physics of Solids, vol. 24, no. 2-3, pp. 125-136, 1976.

(23) M. Rossow, and J. Taylor, "A finite element method for the optimal design of variable thickness sheets," Aiaa Journal, vol. 11, no. 11, pp. 1566-1569, 1973. K.-T. Cheng, and N. Olhoff, "An investigation concerning optimal design of solid elastic plates," International Journal of Solids and Structures, vol. 17 , no. 3, pp. 305-323, 1981.

(25) M. P. Bendsøe, "Optimal shape design as a material distribution problem," Structural and multidisciplinary optimization, vol. 1, no. 4, pp. 193-202, 1989.

(26) J. Sirohi, and I. Chopra, "Fundamental understanding of piezoelectric strain sensors," Journal of intelligent material systems and structures, vol. 11, no. 4, pp. 246-257, 2000.

(27) M. P. Bendsøe, and N. Kikuchi, "Generating optimal topologies in structural design using a homogenization method," Computer methods in applied mechanics and engineering, vol. 71, no. 2, pp. 197-224, 1988.

(28) J. P. Tardivo, A. Del Giglio, C. S. de Oliveira, D. S. Gabrielli, H. C. Junqueira, D. B. Tada, D. Severino, R. de Fátima Turchiello, and M. S. Baptista, "Methylene blue in photodynamic therapy: from basic mechanisms to clinical applications," Photodiagnosis and photodynamic therapy, vol. 2, no. 3, pp. 175-191, 2005.

(29) K. König, V. Bockhorn, W. Dietel, and H. Schubert, "Photochemotherapy of animal tumors with the photosensitizer methylene blue using a krypton laser," Journal of cancer research and clinical oncology, vol. 113, no. 3, pp. 301-303, 1987.

(30) S. M. Ali, H. Y. A.-T. Eman, and S. A. Rajaa, "Photodynamic Therapy for Leiomyosarcoma: In vitro study, Iaqi Journal of Laser, vol. 11, no. B, pp. 1-7, 2012.

(31) J. L. Ferracane, Materials in dentistry: principles and applications: Lippincott Williams \& Wilkins, 2001. 